\title{
A multiscale modified minimum spanning forest method for spatial-spectral hyperspectral images classification
}

\author{
Fereshteh Poorahangaryan ${ }^{1}$ and Hassan Ghassemian ${ }^{2 *}$
}

\begin{abstract}
This paper aimed to present a new method for the spectral-spatial classification of hyperspectral images, based on the idea of modified minimum spanning forest (MMSF). MMSF works on the obtained regions of pre-segmentation step that are considered as nodes of an image graph. In the proposed method, the image is first smoothed by the multiscale edge-preserving filter (MSEPF) and then the MMSF is built in each scale. Finally, all the classification maps of each scale are combined with a majority vote rule. The suggested method, named as MSEPF-MMSF, is performed on four hyperspectral images with different properties, and the experiments deal with the impacts of parameters of filter and the number of markers. The results demonstrate that the proposed method has improved the classification accuracies with respect to the previous methods.
\end{abstract}

Keywords: Edge-preserving filter, Hyperspectral images, Segmentation, Spectral-spatial classification, Minimum spanning forest

\section{Introduction}

Image processing and video analysis are one interesting topic in intelligent systems in recent years [1-3]. In hyperspectral images, a vector with several hundreds of spectrums is assigned to each spatial position. Such numbers are tantamount to the reflexive light spectrum. Such precious spectral information enhances the capability of recognizing physical material and other different objects. One of the valuable uses of hyperspectral images analysis is classification process through which a class is assigned to each image pixel. Hyperspectral image classification represents a hot research area [4-6]. However, the fundamental problems are high dimension and limitation in number of training samples. In order to tackle the problems, different dimension reduction methods [7-9] have been suggested. Extensive researches have been carried out into hyperspectral image classification, most of which only use spectral information. Methods such as neural networks [10], decision tree [11], kernel-based methods

\footnotetext{
* Correspondence: ghassemi@modares.ac.ir

${ }^{2}$ Image Processing and Information Analysis Lab. Faculty of Electrical and Computer Engineering, Tarbiat Modares University, Jalale Ale Ahmad Highway, P.O. Box 14115-111, Tehran, Iran

Full list of author information is available at the end of the article
}

[12], and support vector machine (SVM) [13] have been proposed in order to fulfill the goal. SVM works very well, specifically to limited training samples [13]. Image spatial resolution has been enhanced thanks to advances in the technology of hyperspectral image equipment [14-24]. Therefore, if spatial information (the information obtained from pixel neighborhood) combined with spectral information, classification accuracy will increase. As a result, several numbers of spatial-spectral classification techniques have been introduced in recent years. In general, spatial-spectral classification methods can be divided into two groups. The first group is composed of methods based on a crisp neighborhood system such as the Markov random field (MRF) [14] and related generalized methods $[15,16]$. These methods have achieved appropriate performance in classification. But if neighborhood pixels strongly depended on each other, the concept of neighborhood may not include effective samples. On the other hand, using a large neighborhood may cause computational problems [17]. The second group is composed of methods based on adaptive neighborhood. The morphology-based methods [18] and the segmentation-based methods [19-24] fall in this category. Although morphology-based methods are 
instrumental tools for obtaining spatial information, the shape of the structuring element (SE) and computational complexity are still key issues in such classifications [18]. Segmentation is also a powerful tool for defining spatial dependency where, by this tool, an image can be divided into homogenous regions. Segmentation techniques extract a large neighborhood for large regions. Meanwhile, small regions containing one or more pixels are not eliminated. Methods like watershed [19], clustering [20], hierarchical segmentation [21], and marker-controlled segmentation methods $[22,23]$ have been presented for segmentation and classification of hyperspectral images. Meanwhile, marker-controlled segmentation methods such as SVM-MSF (SVM-minimum spanning forest) [22] and MSSC-MSF (multispectral-spatial classifier-MSF) [23] can decrease over-segmentation and increase classification accuracy more than other methods [21]. The SVM-MSF uses probability SVM and consequently MSF. The MSSC-MSF uses multispectral-spatial classification for marker selection, and MSF is built on the obtained markers. However, marker selection is still a fundamental challenge. In addition, applying an extra step for marker selection leads to an increase in computation time.

Spatial filtering has been recently used in researches into hyperspectral image processing and has shown its efficiency in this topic $[24,25]$. The simplicity of understanding this type of filters is their main advantage, and their complexity are low [24]. Filters like weighted mean filter (WMF) [24] and edge-preserving filter (EPF) [25] are utilized to reduce image noise, increase consistency of neighborhood pixels, and provide homogenous regions.

In this paper, we propose a combination of spatial filter and MSF segmentation method for spatial-spectral hyperspectral image classification. In most of the researches related to hyperspectral image processing, single-scale filtering has been taken into use. But single-scale spatial filters have no capability to describe different spatial structure of the hyperspectral images. Therefore, a multiscale edge-preserving filter has been proposed and used in the mentioned hybrid scheme. Furthermore, a modified model of MSF has been proposed and used in the suggested scheme. Despite traditional MSF that operates at the pixel scale, our model operates at the regional scale, and this process leads to a decrease in computational time.

\section{Multiscale edge-preserving filter}

Gaussian filtering is one of the most common methods for smooth an image. In this method, the underlying assumption is that images change slowly over space. On the other hand, the assumption does not work at edges. Despite Gaussian filters, EPF preserves the edges. In order to cope with this issue, the bilateral filter as one type of EPFs can be used. The bilateral filter has had wide applications thanks to the advantage of preserving edges up to now. There are two conditions where the pixels can be close to each other. They can have a nearby spatial location, or they have a similar spectral signature. The traditional filtering is a spatial domain filtering. The combination of both the spatial domain and range filtering is known as bilateral filtering.

Since the hyperspectral images may consist of information in different resolutions, one scale of the filtering cannot extract the significant information [25]. To overcome this challenge, we present a multiscale edge-preserving filter called MSEPF. The proposed MSEPF (denoted as $\mathrm{H}$ ) is defined as follows:

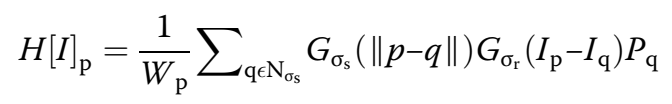

with

$$
\begin{aligned}
& N_{\sigma_{\mathrm{s}}}=\left(2 \sigma_{\mathrm{s}}+1\right) \times\left(2 \sigma_{\mathrm{s}}+1\right) \quad \sigma_{\mathrm{s}}=1,2, \ldots, Q . \\
& W_{\mathrm{p}}=\sum_{\mathrm{q} \in \mathrm{N}_{\sigma_{\mathrm{s}}}} G_{\sigma_{\mathrm{s}}}(\|p-q\|) G_{\sigma_{\mathrm{r}}}\left(I_{\mathrm{p}}-I_{\mathrm{q}}\right)
\end{aligned}
$$

where $p$ and $q$ represent the $p$-th and $q$-th pixels; $P$ and $I$ are the input image and reference image, respectively; $\sigma_{\mathrm{s}}$ denotes the $\sigma_{\mathrm{s}}$-th level (scale) of the decomposition; $Q$ is the number of the scale; $N_{\sigma_{\mathrm{s}}}$ is a local window of size $\left(2 \sigma_{\mathrm{s}}+1\right) \times\left(2 \sigma_{\mathrm{s}}+1\right)$ at the $\sigma_{\mathrm{s}}$-th scale around pixel $p$; and $W_{\mathrm{p}}$ is a normalization factor. $G_{\sigma_{\mathrm{s}}}$ and $G_{\sigma_{\mathrm{r}}}$ can be obtained from Eqs. (4) and (5), respectively.

$$
\begin{aligned}
& G_{\sigma_{\mathrm{s}}}(\|p-q\|)=\exp \left(-\frac{\|p-q\|}{\sigma_{\mathrm{s}}^{2}}\right) \\
& G_{\sigma_{\mathrm{r}}}\left(I_{\mathrm{p}}-I_{\mathrm{q}}\right)=\exp \left(-\frac{\left(I_{\mathrm{p}}-I_{\mathrm{q}}\right)^{2}}{\sigma_{\mathrm{r}}^{2}}\right)
\end{aligned}
$$

where the vectors $p$ and $q$ epitomize the spatial location of the image. Furthermore, $I_{\mathrm{p}}$ and $I_{\mathrm{q}}$ are the intensity values of the locations $p$ and $q$, respectively, and $P$ and $I$ are the input image and the reference image, respectively. $G_{\sigma_{\mathrm{s}}}$ is a spatial Gaussian function. The more the distance from the center, the less $G_{\sigma_{\mathrm{s}}}$ becomes. $G_{\sigma_{\mathrm{r}}}$ is a range Gaussian function. According to Eq. (4), the increase in the intensity difference between $I_{\mathrm{p}}$ and $I_{\mathrm{q}}$ leads to the decrease in $G_{\sigma_{\mathrm{r}}}$. Parameters $\sigma_{\mathrm{s}}$ and $\sigma_{\mathrm{v}}$ which are the standard deviations of Gaussian functions $G_{\sigma_{\mathrm{s}}}$ and $G_{\sigma_{\mathrm{r}}}$, respectively, determine the amount of filtering for the image $I$ [25].

\section{Modified minimum spanning forest}

For constructing a minimum spanning forest (MSF), a Bband hyperspectral image is given, which can be considered as a set of $n$-pixel vectors $X=\left\{x_{\mathrm{j}} \in R_{\mathrm{B}}, j=1,2, \ldots, \mathrm{n}\right\} . V$ and $E$ are the sets of vertices and edges, respectively, and $W$ is a mapping of the set of edges $E$ into $R^{+}$in the undirected graph $G=(V, E, W)$. Per pixel of image is regarded as 


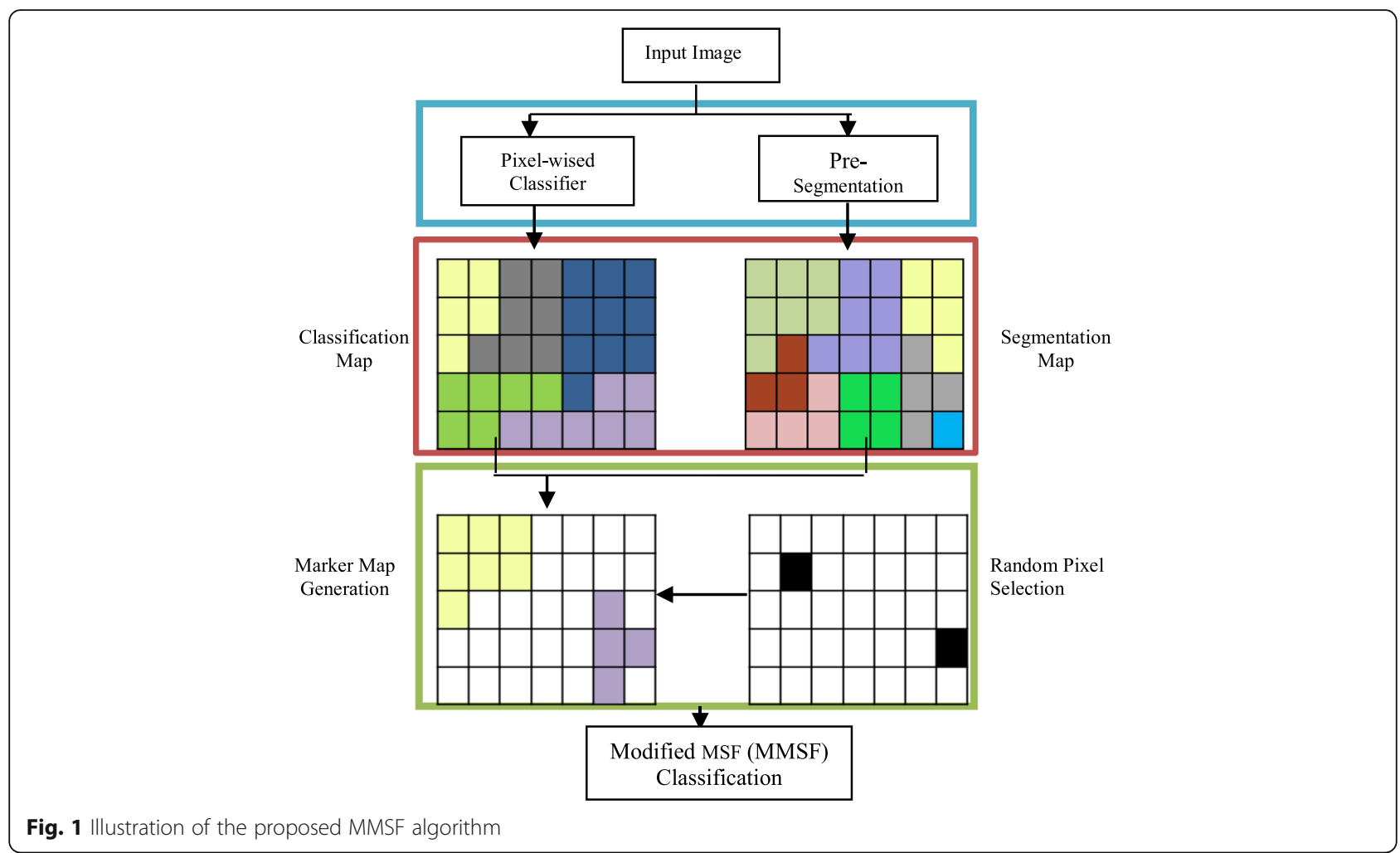

a vertex $v \in V$ and each edge $e_{\mathrm{ij}} \in E$ connects two vertices $i$ and $j$ to each other. A non-zero weight $w_{\mathrm{i}, j}$ is assigned to each edge. In order to compute weights of edges, spectral angle mapper (SAM) and L1 vector norm can be used [22]. Generation of an MSF on an image is performed as the following steps:

- Convert image to graph according to the abovementioned way.

- Compute weights of edges.

- Add additional vertices $t_{\mathrm{i}}, i=1, \ldots, m$, corresponding to $m$-selected markers.
- Link per additional vertex $t_{\mathrm{i}}$ to the pixels representing a marker $i$ by the zero-weighted edge and construct a new graph [22].

An MSF in $G$ is included in the minimum spanning tree of the graph, and every tree is grown on a vertex $t_{\mathrm{i}}$; when the vertex $r$ is taken away, the MSF is produced. Prim's algorithm is employed for generating the MSF; for more details, please see [22].

The modified MSF (MMSF) as a part of the overall flowchart of the proposed spectral-spatial classification method is shown in Fig. 1. As shown in Fig. 1,

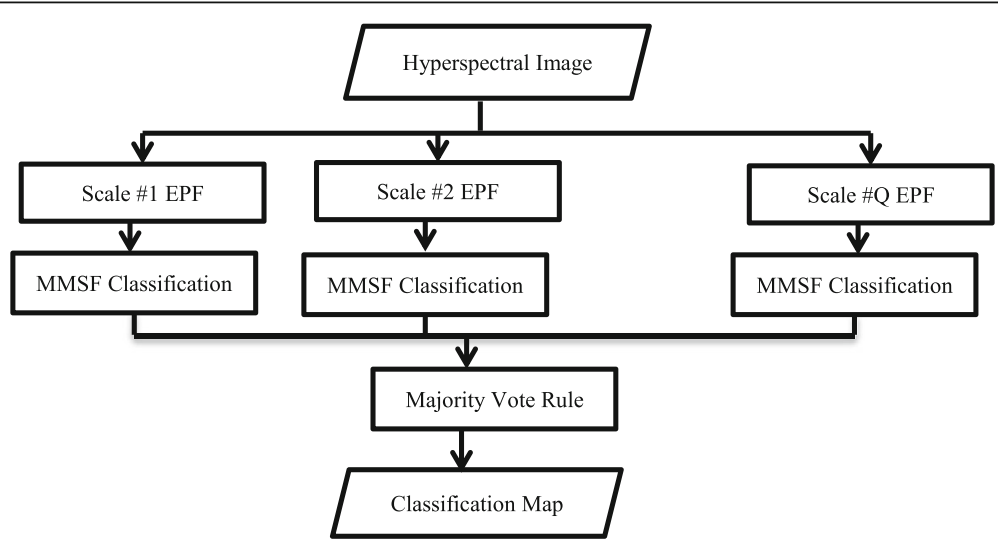

Fig. 2 Flowchart of the proposed spectral-spatial classification method (MSEPF-MMSF) 


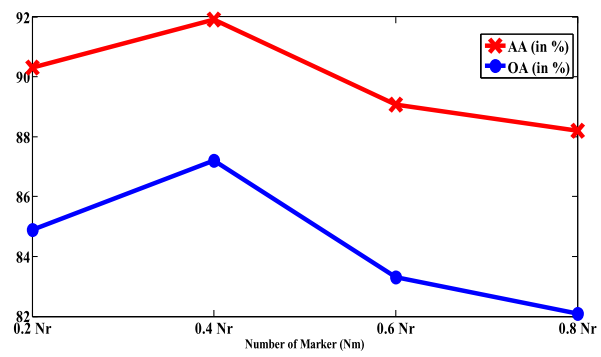

(a)

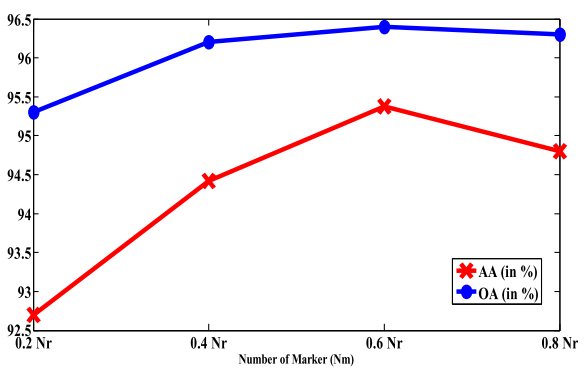

(b)

Fig. 3 Change of the $O A$ and $A A$ against the number of $N_{m}$. a Indian Pines image. b PaviaU image

the image is firstly pre-segmented. So, the image is segmented to many small regions regarded as the nodes of MMSF. Compared to traditional MSF, the MMSF algorithm is presented in a smaller number of nodes and edges. On the other hand, a pixel-wised classification is performed. After the mentioned steps, $N_{\mathrm{m}}$ pixels are chosen randomly. One region is specified for each selected pixel based on the presegmentation map. Subsequently, all the pixels of a region are appointed to the class with the highest frequency according to the result of the pixel-wised classifier in the given region. These regions are regarded as markers for MMSF construction. Also, a segmentation $S$ is a division of $V$ into regions such that each region $R \in S$ accords with a connected component in a graph $G^{\prime}=\left(V, E^{\prime}\right)$, where $E^{\prime} \in E$. The differences between the two regions $R_{1}, R_{2} \subset V$ can be obtained by the minimum weight edge linking the two regions. That is,

$$
\begin{aligned}
& w\left(R_{1}, R_{2}\right)=\min \quad w\left(v_{\mathrm{i}}, v_{\mathrm{j}}\right) \\
& v_{\mathrm{i}} \in R_{1}, v_{\mathrm{j}} \in R_{2},\left(v_{\mathrm{i}}, v_{\mathrm{j}}\right) \in E \\
& \text { and } w\left(v_{\mathrm{i}}, v_{\mathrm{j}}\right)=w_{\mathrm{ij}}
\end{aligned}
$$

$w\left(R_{1}, R_{2}\right)=\infty$ is allowed if no edge links $R_{1}$ and $R_{2}$. According to this measure, the algorithm will combine two regions even though there is an edge with a low weight between them.

\section{Proposed spectral-spatial classification method}

This section introduces a new spectral-spatial classification scheme for hyperspectral images. The overall flowchart of the proposed spectral-spatial classification method (MSEPF-MMSF) is given in Fig. 2.

The MSEPF-MMSF is composed of the following three main steps: (1) preprocessing using MSEPF, (2) spatialspectral classification of each individual scale of filtering using MMSF, and (3) majority vote rule for class decision.

There is a B-band hyperspectral image $X=\left\{\boldsymbol{x}_{\mathrm{j}} \in R_{\mathrm{B}}\right.$, $j=1,2, \ldots, n\}$ at the input. At first, the hyperspectral image is smoothed by multiscale bilateral filter defined in Section 2. At this point, the controversial issue is the reference image selection, $I$. In order to tackle this problem, principal component analysis (PCA) is applied because the optimum portrayal of the image is presented in the mean-squared sense. Original hyperspectral image undergoes dimension reduction via PCA, and the first $r$ principal components are chosen as the reference image for the bilateral filter. The value of $r$ can be automatically selected by analyzing the eigenvalues of scatter matrices computed from the PCA [26]. In this step, we have Q-filtered images. A spectral-spatial classification using MMSF is done in each individual $Q$ scale. We propose to use an

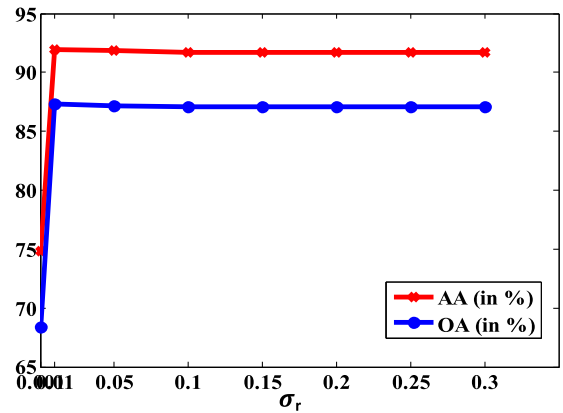

(a)

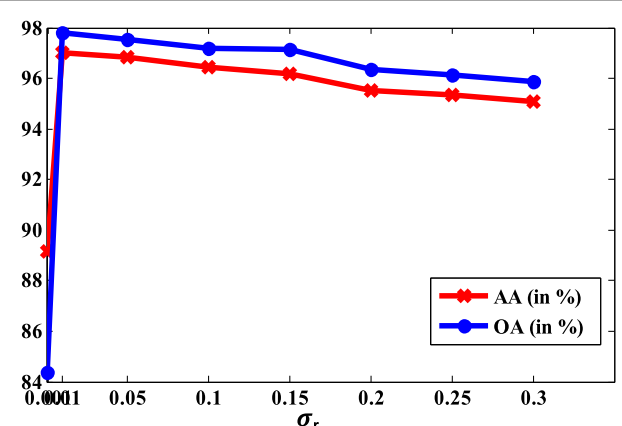

(b)

Fig. 4 Analysis of the influence of the parameter $\sigma_{r}$. a Indian Pines image. b PaviaU image 


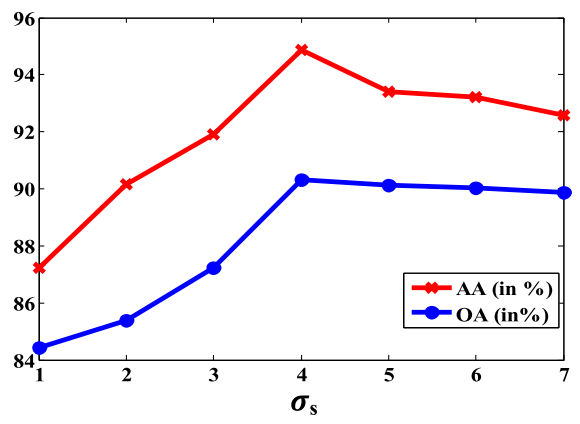

(a)

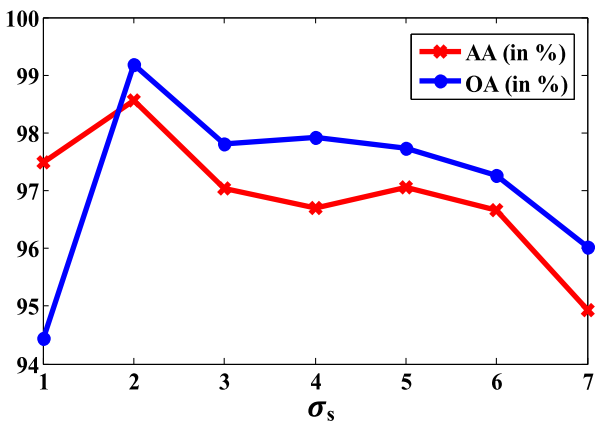

(b)

Fig. 5 Analysis of the influence of the parameter $\sigma_{s}$. a Indian Pines image. b PaviaU image

SVM classifier for pixel-wised classification, which is strongly suitable for hyperspectral data classification [13]. In the pre-segmentation stage, we implemented watershed since it detects good boundaries and retains small differences within the objects. Typically, the watershed transformation is used for a one-band image. There are several ways for generalizing this technique to an r-band hyperspectral image [19].

In the final step, the classification maps of each individual scale are combined with a maximum vote decision rule and the final classification map is obtained. At this step of the algorithm, there are $Q$ spectral-spatial classification maps corresponding to the $Q$ scale of the filtering step. The majority vote procedure is described in the following way: For each pixel, a $1 \times Q$ vector is considered based on assigned classes in $Q$ spectralspatial classification maps. In addition, the pixel is allocated to the highest frequency class. Every pixel of the image undergoes the abovementioned procedure. So the final classification map is acquired.

\section{Results and discussion}

The experimental study is done to indicate the performance of the proposed spectral-spatial classification scheme. In this section, the experimental data sets are introduced and two various experiments are explained.

Table 1 Information classes, number of training samples, and classification accuracy for the Indian Pines

\begin{tabular}{|c|c|c|c|c|c|}
\hline & No. of samples & No. of training samples & SVM & SSEPF-MMSF & MSEPF-MMSF \\
\hline Alfalfa & 46 & 15 & 95.65 & 100 & 100 \\
\hline Corn-notill & 1428 & 50 & 64.85 & 80.31 & 86.55 \\
\hline Corn-mintill & 830 & 50 & 61.57 & 91.81 & 90.72 \\
\hline Corn & 237 & 50 & 86.08 & 98.73 & 100 \\
\hline Grass-pasture & 483 & 50 & 92.55 & 95.86 & 94.62 \\
\hline Grass-trees & 730 & 50 & 87.81 & 98.36 & 97.95 \\
\hline Grass-pasture-mowed & 28 & 15 & 96.43 & 96.43 & 100 \\
\hline Hay-windrowed & 478 & 50 & 99.16 & 100 & 100 \\
\hline Oats & 20 & 15 & 100 & 100 & 100 \\
\hline Soybean-notill & 972 & 50 & 66.46 & 87.74 & 88.79 \\
\hline Soybean-mintill & 2455 & 50 & 47.94 & 84.95 & 85.38 \\
\hline Soybean-clean & 593 & 50 & 71.5 & 93.57 & 92.22 \\
\hline Wheat & 205 & 50 & 99.02 & 100 & 100 \\
\hline Woods & 1265 & 50 & 83.32 & 96.13 & 97.15 \\
\hline Buildings-G-T-D & 386 & 50 & 71.76 & 99.22 & 98.96 \\
\hline Stone-steel-towers & 93 & 50 & 91.4 & 100 & 100 \\
\hline AA (\%) & - & - & 82.21 & 94.87 & 95.64 \\
\hline $\mathrm{OA}(\%)$ & - & - & 69.79 & 90.31 & 91.45 \\
\hline Kappa coefficient & - & - & 66.27 & 89.18 & 90.32 \\
\hline
\end{tabular}


Table 2 Information classes, number of training samples, and classification accuracy for the PaviaU

\begin{tabular}{llllll}
\hline & No. of samples & No. of training samples & SVM & SSEPF-MMSF & MSEPF-MMSF \\
\hline Asphalt & 6631 & 548 & 82.17 & 99.14 & 99.2 \\
Meadows & 18,649 & 540 & 87.25 & 97.28 & 99.23 \\
Gravel & 2099 & 392 & 81.23 & 95.95 & 95.43 \\
Trees & 3064 & 524 & 93.21 & 98.66 & 96.28 \\
Metal sheets & 1345 & 265 & 99.78 & 99.93 & 100 \\
Bare soil & 5029 & 532 & 91.05 & 99.54 & 99.8 \\
Bitumen & 1330 & 375 & 89.4 & 99.25 & 900 \\
Bricks & 3682 & 514 & 85.17 & 97.66 & 98.18 \\
Shadows & 947 & 231 & 99.89 & 99.79 & 97.36 \\
AA (\%) & - & - & 89.9 & 98.57 & 98.36 \\
OA (\%) & - & - & 87.6 & 99.18 & 98.8 \\
Kappa coefficient (\%) & - & - & 85.23 & 98.81 & 98.41 \\
\hline
\end{tabular}

In the first experiment, two well-known data sets including the non-urban data set (e.g., Indian Pines) and urban data set (e.g., PaviaU) are used. The effectiveness of parameters $\left(N_{\mathrm{m}}, \sigma_{\mathrm{r}}, Q\right)$ of the proposed method is analyzed, and their optimal values are extracted. Also, the classification results and comparison with other methods are given. In the second experiment, the proposed method using the extracted optimal values is applied on two other data sets including Botswana and SalinasA. In this experiment, it is demonstrated that the good classification accuracies for various images will be achieved with the presented parameter setting. In addition, the classification results and discussion are presented.

\subsection{Data sets}

Four well-known hyperspectral data sets, i.e., the Indian Pines, the University of Pavia, the Salinas, and Botswana, go under the classification approach. The Indian Pines image capturing the agricultural Indian Pine test site of north-western Indiana was recorded by the AVIRIS sensor. This image contains 220 bands ranging from 0.4 to $2.5 \mu \mathrm{m}$ with a spatial resolution of $20 \mathrm{~m}$ per pixel. It consists of $145 \times 145$ pixels and contains 16 classes. Twenty water absorption bands were removed before experiments (the removed bands are [104-108], [150-163], and 220). The ROSIS-03 satellite sensor captured an image of an urban area surrounding the University of $\mathrm{Pa}$ via. This image is called PaviaU. The image is with the size $610 \times 340$ pixels and has 115 bands ranging from 0.43 to $0.86 \mu \mathrm{m}$ with a spatial resolution of $1.3 \mathrm{~m}$ per pixel. In addition, the 12 most noisy channels were removed before experiments. Nine classes are considered for this image. The Salinas image was recorded by the AVIRIS sensor over Salinas Valley, CA, USA. This data set has 224 bands in the wavelength range $0.4-2.5 \mu \mathrm{m}$ with the size $512 \times 217$. The bands of [108-112], [154-167], and 224 are eliminated because of the water absorption bands, and
204 bands with a spatial resolution of $3.7 \mathrm{~m}$ per pixel are applied in our study. A subset of this image, named as SalinasA, is used. It is with the size $86 \times 83$ pixels, located in the [591-678] $\times$ [158-240] of Salinas Valley, containing six classes. The Botswana data set was recorded by the Hyperion sensor over Okavango Delta, Botswana. It contains 242 bands covering the $400-2500 \mathrm{~nm}$ with a spatial resolution of $30 \mathrm{~m}$ per pixel. The noisy bands ([10-55, 82-97, 102-119, 134-164, 187-220]) were removed, and the remaining 145 bands were used for experiments. This data set consists of 14 identified classes.

\subsection{First experiment}

In the used filters, the parameters $\sigma_{\mathrm{s}}$ and $\sigma_{\mathrm{r}}$ represent the filtering size and blur degree, respectively. In addition, the numbers of $N_{\mathrm{m}}$ influence the classification accuracy. In order to indicate the influence of $N_{\mathrm{m}}$, four

Table 3 Average accuracy (AA), overall accuracy (OA), and kappa for some segmentation-based and filter-based methods on Indian Pines and Pavia University data sets

\begin{tabular}{|c|c|c|c|c|c|c|c|}
\hline \multirow[t]{2}{*}{ Methods } & \multirow[t]{2}{*}{ Ref. } & \multicolumn{3}{|c|}{ Indian Pines } & \multicolumn{3}{|c|}{ PaviaU } \\
\hline & & $\mathrm{AA}$ & $\mathrm{OA}$ & Kappa & $\mathrm{AA}$ & $\mathrm{OA}$ & Kappa \\
\hline SVM-MSF & {$[22]$} & 91.06 & 88.4 & 87 & 84.14 & 92.3 & 80 \\
\hline MSSC-MSF & {$[23]$} & 92.3 & 94.2 & 91 & 98.6 & 97.9 & 97 \\
\hline RD-MSF & {$[24]$} & 93.73 & 91.33 & - & - & - & - \\
\hline Pixon based & {$[6]$} & 92.18 & 89.94 & 88 & 94.72 & 95.17 & 94 \\
\hline SVMMRF-HSRM & {$[5]$} & - & 93.10 & 92.11 & - & - & - \\
\hline MLRsubMLL-HSRM & {$[5]$} & - & 80.21 & 82.60 & - & - & - \\
\hline EPF & {$[25]$} & 94.46 & 90.28 & 89.98 & 98.38 & 99.09 & 98.77 \\
\hline MSF-KNN & {$[29]$} & 90.1 & 88.67 & 87.32 & 94.39 & 95.1 & 94.12 \\
\hline AMG-M-HSEG & {$[30]$} & 94.83 & 91.4 & 89.48 & 97.18 & 97.45 & 97.23 \\
\hline $\begin{array}{l}\text { MSEPF-MMSF } \\
\text { (Proposed method) }\end{array}$ & - & 95.64 & 91.45 & 90.32 & 98.36 & 98.8 & 98.41 \\
\hline
\end{tabular}




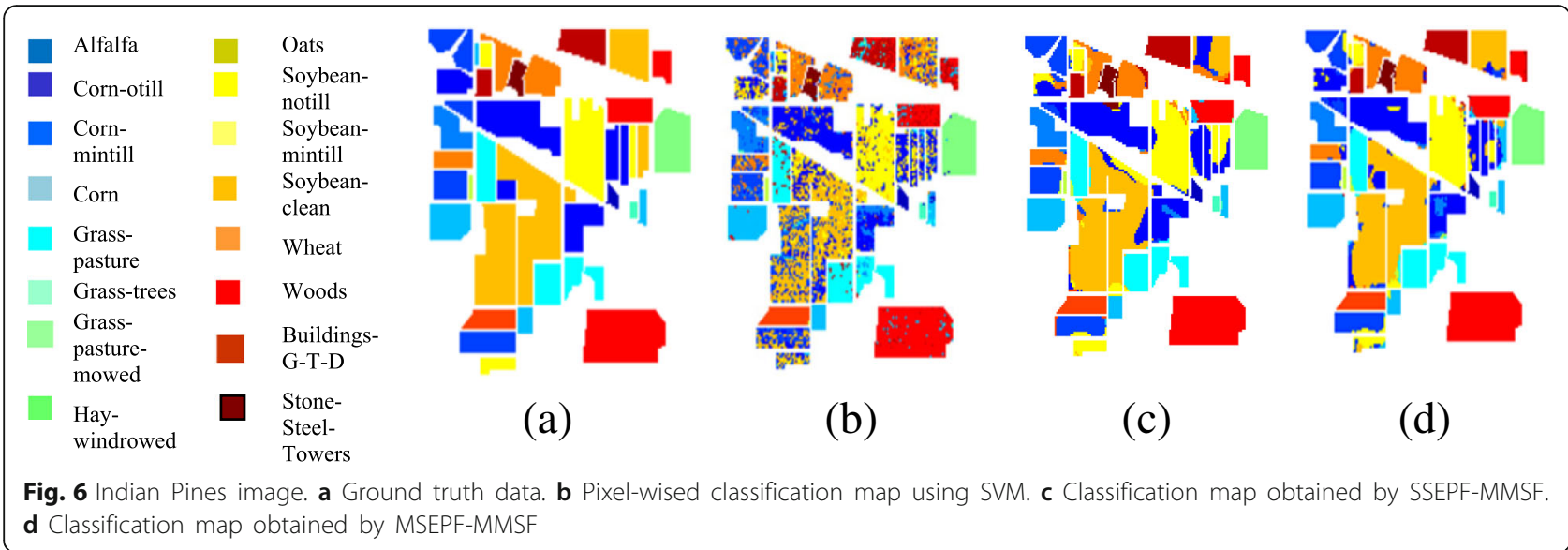

different values have been determined, i.e., $0.2 N_{r}, 0.4 N_{\mathrm{r}}$ $0.6 N_{\mathrm{r}}$, and $0.8 N_{\mathrm{r}}$.

$N_{\mathrm{r}}$ symbolizes the number of regions in the segmentation map obtained from the watershed. While the impact of analysis of the $N_{\mathrm{m}}$ is being analyzed, $\sigma_{\mathrm{r}}$ and $\sigma_{\mathrm{s}}$ are supposed to be 0.2 and 3 , respectively. The evolution of the overall accuracy (OA) and average accuracy (AA) against the $N_{\mathrm{m}}$ for Indian Pines and PaviaU data set is presented in Fig. 3. With an increase in $N_{\mathrm{m}}$, the classification accuracies increase until a zenith point is obtained. Reaching the maximum amount, the classification accuracies initiate to reduce up to the SVM results. This figure shows that the highest amount of accuracy values is obtained when $N_{\mathrm{m}}$ is $0.4 N_{\mathrm{r}}$ and $0.6 N_{\mathrm{r}}$ for the two abovementioned data sets, respectively. Although astounding, this difference of optimal value for $N_{\mathrm{m}}$ can be explained. In the non-unban data set, e.g., Indian Pines image, the objects are pretty wide and usually well delimited. So, though it is not high, the number of $N_{\mathrm{m}}$ will be a fairly accurate classification. However, the PaviaU image includes a lot of small regions. Thus, there should be enough number of $N_{\mathrm{m}}$ in order to statistically have more opportunity to get at least one marker in each various region of the image. In conclusion, there is a higher amount of optimal value for $N_{\mathrm{m}}$ compared to the other images.

The impact of $\sigma_{\mathrm{r}}$ and $\sigma_{\mathrm{s}}$ on the classification efficiency is investigated in Figs. 4 and 5, respectively. $N_{\mathrm{m}}$ is fixed to be the optimal value and $\sigma_{\mathrm{s}}$ equals 3 during the analysis of the effect of $\sigma_{\mathrm{r}}$. In Fig. 4a, b, we can see the evolution of the AA and OA against $\sigma_{\mathrm{r}}$. Seven different modes, i.e., $1,2, \ldots, 7$, are considered so as to show the influence of $\sigma_{\mathrm{s}}$. Figure $5 \mathrm{a}$, b shows the changes of classification accuracies as a function of $\sigma_{\mathrm{s}}$ for two data.

From Fig. 5, it is concluded that the optimal values of $\sigma_{\mathrm{s}}$ are 4 and 2 for the Indian Pines and PaviaU images, respectively. The results support that these parameters cannot be too small or too large. If the filtering size and blur degree, i.e., $\sigma_{\mathrm{s}}$ and $\sigma_{\mathrm{r}}$ are too large, there may be a dramatic decrease in the average classification accuracy that may over-smooth the maps. So, those small-scale objects may be misclassified. Likewise, a very small filtering size or blur degree is also unsatisfactory for the

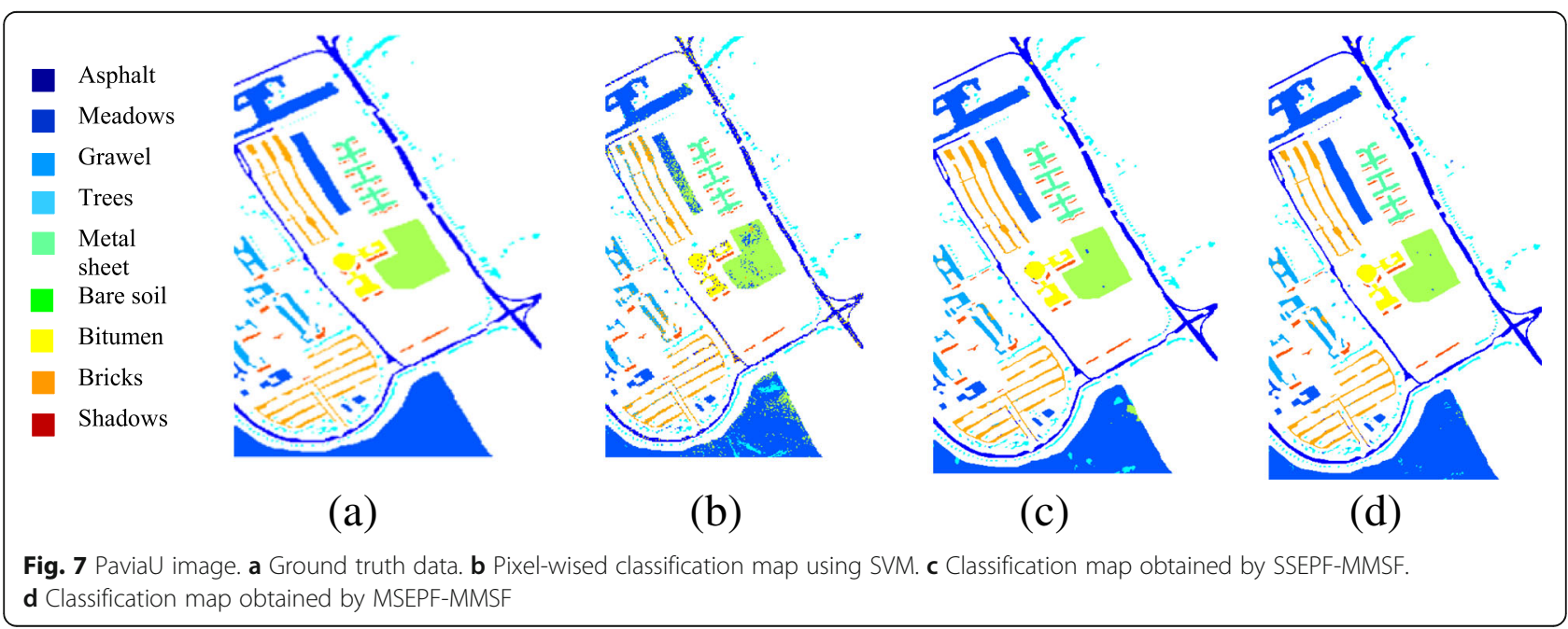




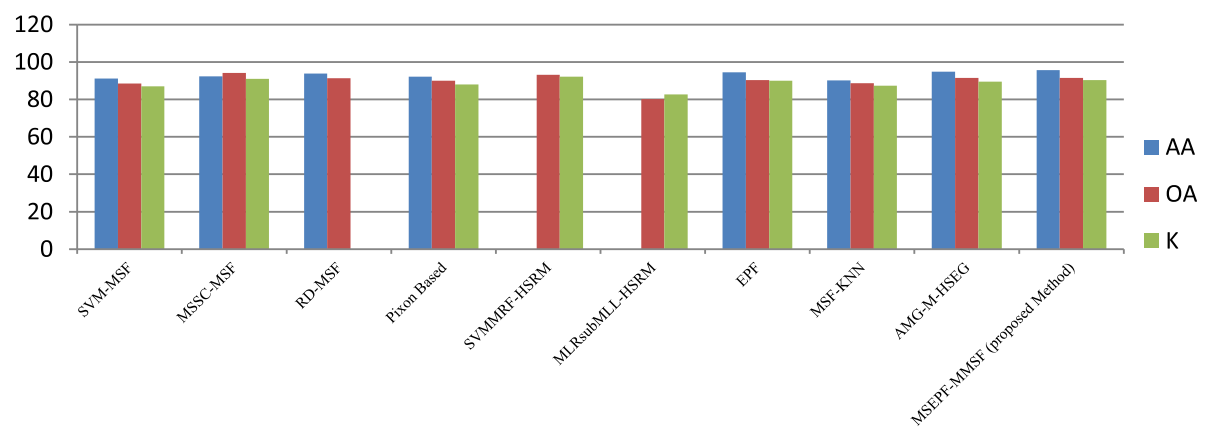

Fig. 8 Comparison between the proposed classifier and some state-of-the-art methods for Indian Pines

proposed method since it connotes that only very limited local spatial information is used in the filtering process. Hereafter, the optimal values of $\sigma_{\mathrm{r}}$ and $N_{\mathrm{m}}$ are employed in each image. In our previous method [27], single-scale edge-preserving filter (SSEPF) is applied. Then, spatial-spectral classification is constructed using MMSF. Thus, this method is called SSEPF-MMSF. In SSEPF-MMSF, we used optimal values of both of $\sigma_{s}$ and $\sigma_{\mathrm{p}}$ which are obtained from the experiment. In MSEPFMMSF (this work), seven values from 1 to 7 of $\sigma_{\mathrm{s}}$ are used and construction of MMSF is repeated for each scale. The final classification label for each pixel is achieved by combining the multiple results via majority voting.

Tables 1 and 2 present the classification results for the two mentioned data sets. These tables show the common classification indexes, e.g., average accuracy (AA), overall accuracy (OA), and kappa coefficient. Furthermore, the classification accuracy of each class obtained by pixel-wised classifier and proposed methods are given. In these tables, the results of Monte Carlo simulations (after 10 runs) are reported. Training samples were randomly selected from the Ground truth data.

It can be understood that through using the proposed classifier, the classification accuracies are increased significantly when compared to the pixel-wised classification. In the Indian Pines, the MSEPF-MMSF increases the classification accuracy compared to the SVM classifier by about
$13 \%$. In the PaviaU data set, the OA is improved by $18 \%$ points and the AA by $12.13 \%$ points. It is worth noting that the SSEPF-MMSF classifier is presented with better performance than the MSEPF-MMSF classifier in the PaviaU. In the following, the reason for this result is explained. PaviaU data set contained many small areas such as shadow.

As can be seen from Table 3, the MSEPF-MMSF improves all of the accuracies than SSEPF-MMSF except for the classes shadows and trees. These classes contained the very small object. Furthermore, at the small scale of filtering, the SSEPF-MMSF has been proven to be more suitable for classification of such classes. Increasing the scale of filtering may over-smooth the maps, and thus, such classes may be misclassified. In the MSEPF-MMSF method, the results of different scales are combined using majority voting. Because these classes are not on a large scale with good results, the classification accuracy of the MSEPF-MMSF method is lower than that of SSEPFMMSF. As a result, the MSEPF-MMSF might not be the most suitable one for classifying such images that contain very small objects. Considering the obtained results, it is clear that the final classification results are the best ones although the initial pixel-wised classifier does not perform very well. The adjacent pixel consistency increases and provides more homogeneous objects brought on by the filtering step. Thus, much smoother classification maps

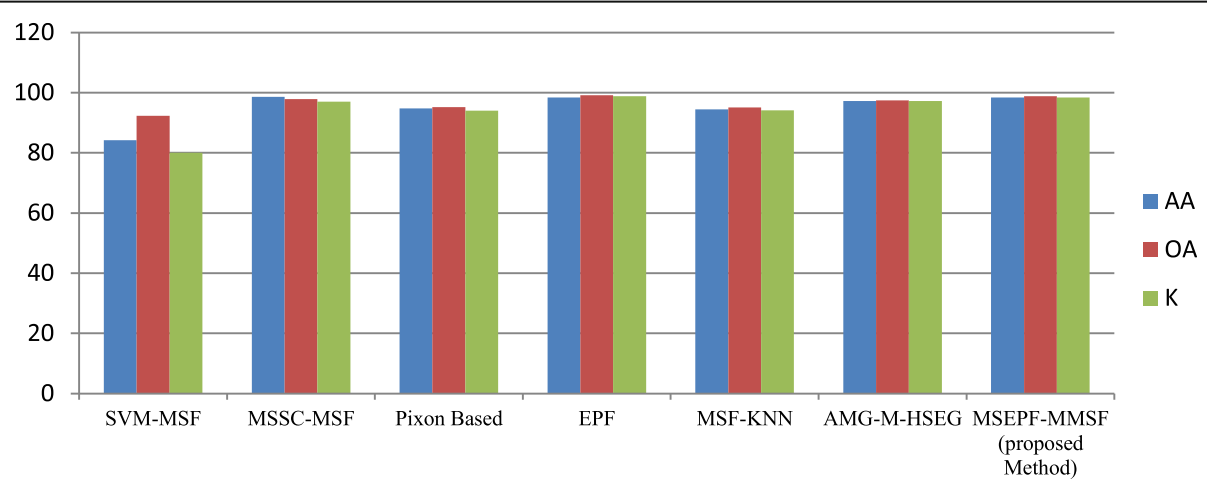

Fig. 9 Comparison between the proposed classifier and some state-of-the-art methods for PaviaU 
Table 4 Information classes, number of training samples, and classification accuracy for the SalinasA

\begin{tabular}{llllll}
\hline & No. of Samples & No. of training samples & SVM & SSEPF-MMSF & MSEPF-MMSF \\
\hline Brocoli_green_weeds_1 & 391 & 6 & 99.49 & 100 & 100 \\
Corn_senesced_green_weeds & 1343 & 6 & 97.69 & 97.54 & 98.98 \\
Lettuce_romaine_4wk & 616 & 6 & 93.67 & 99.68 & 98.78 \\
Lettuce_romaine_5wk & 1525 & 6 & 99.93 & 99.8 & 100 \\
Lettuce_romaine_6wk & 674 & 6 & 99.11 & 99.85 & 100 \\
Lettuce_romaine_7wk & 799 & 6 & 96.62 & 100 & 100 \\
AA (\%) & - & - & 97.75 & 99.47 & 99.62 \\
OA (\%) & - & - & 96.59 & 99.4 & 99.48 \\
Kappa coefficient (\%) & - & - & 96.33 & 99.31 & 99.4 \\
\hline
\end{tabular}

are obtained. The proposed multiscale method can describe different spatial structures, and hence, our proposed algorithm has higher classification accuracy than the SSEPF-MMSF, except for the PaviaU image. It should be noted that the classification results of SSEPF-MMSF and MSEPF-MMSF are so close together for the PaviaU data set. It can be concluded that the SSEPF-MMSF method has been proven to be more adaptable for the classification of the urban area, but the MSEPFMMSF method is suitable for the non-urban area. The Ground truth data, the classification maps of pixel-wised classifier, and our proposed classifiers (SSEPF-MMSF and MSEPF-MMSF) for the two data sets are shown in Figs. 6 and 7. These figures show that the classification map represented by the SVM is not very well because it is still possible to see some noisy pixels, whereas the proposed classifiers provide a smoother classification map.

Although the MSEPF-MMSF method has good performance, the primary problem of this method is determining the optimal values for the parameters $N_{\mathrm{m}}$, $\sigma_{\mathrm{r}}$ and $Q$. Based on the performed experiments, default values can be suggested for these parameters. For $\sigma_{\mathrm{r}}$ it can be shown that with increasing values of $\sigma_{\mathrm{r}}$ the computation time is increased without giving better results. Therefore, we suggest setting this parameter to 0.05 . For $Q$, it has been observed in our experiments that after the seventh scale, there is no significant improvement in the accuracy values. We suggest setting this parameter to 7 . The determination of the optimal value of $N_{\mathrm{m}}$ is not a simple task. It has been indicated that for the images with a big region (such as Indian Pines), the optimal

Table 5 Information classes, number of training samples, and classification accuracy for the Botswana

\begin{tabular}{llllll}
\hline & No. of samples & No. of training samples & SVM & SSEPF-RSMSF & MSEPF-RSMSF \\
\hline Water & 270 & 23 & 100 & 99.63 & 100 \\
Hippo grass & 101 & 23 & 89.11 & 100 & 100 \\
Floodplain grasses1 & 251 & 25 & 100 & 100 & 100 \\
Floodplain grasses2 & 215 & 23 & 93.49 & 100 & 100 \\
Reeds1 & 269 & 23 & 85.5 & 96.65 & 98.88 \\
Riparian & 269 & 23 & 84.76 & 100 & 100 \\
Firescar2 & 259 & 23 & 97.68 & 100 & 100 \\
Island interior & 203 & 23 & 97.04 & 100 & 100 \\
Acacia woodlands & 314 & 24 & 92.04 & 100 & 100 \\
Acacia shrublands & 248 & 23 & 95.56 & 100 & 100 \\
Acacia grasslands & 305 & 23 & 90.49 & 99.67 & 100 \\
Short mopane & 181 & 23 & 90.61 & 100 & 100 \\
Mixed mopane & 268 & 23 & 92.16 & 100 & 100 \\
Exposed soils & 95 & 23 & 100 & 100 & 99.67 \\
AA (\%) & - & - & 93.46 & 99.7 & 99.89 \\
OA (\%) & - & - & 93.23 & 99.66 & 99.63 \\
Kappa coefficient & - & & 92.66 & 99.87 & 9 \\
\hline
\end{tabular}




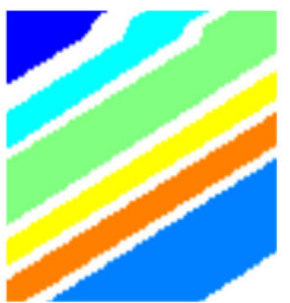

(a)

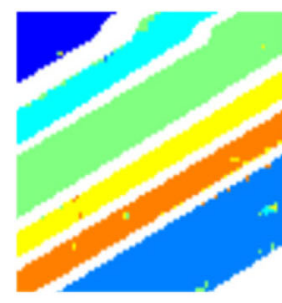

(b)

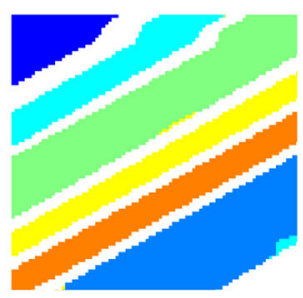

(c)

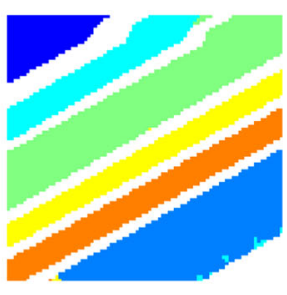

(d)

Fig. 10 SalinasA image. a Ground truth data. b Pixel-wised classification map using SVM. c Classification map obtained by SSEPF-MMSF. d Classification map obtained by MSEPF-MMSF

value of $N_{\mathrm{m}}$ is $40 \%$ of the total number of regions of the pre-segmented image. However, for the image such as PaviaU, which is composed of many small areas, the optimal value of $N_{\mathrm{m}}$ is $60 \%$ of the total number of regions of the pre-segmented image. Thus, the user should have background knowledge of the image in order to obtain the best results.

In Table 3 and Figs. 8 and 9, the proposed classifier is compared with classification results obtained with some state-of-the-art methods for Indian Pines and PaviaU data sets. These methods include construction MSF on markers obtained from probability SVM (SVM-MSF) [22], classification by building an MSF on markers extracted from multispectral-spatial classifier (MSSCMSF) [23], classification by construction of an MSF on random markers (RD-MSF) [28], classification by construction of an MSF on extracted markers of KNN (MSF-KNN) [29], classification by the algebraic multigrid method and hierarchical segmentation algorithm (AMG-M-HSEG) [30], edge-preserving filter (EPF)based classification [25], adaptive pixon extraction technique [6], and two types of hierarchical statistical region merging (HSRM) [5] for Indian Pines and PaviaU data sets. The classification results obtained by different approaches for the Indian Pines data set with 50 random training samples per class (15 for smaller classes) are given. Furthermore, we reported the classification accuracy of the mentioned schemes for PaviaU data set with 3921 total training samples. The MSEPF-MMSF method can describe different spatial structures, and hence, our proposed algorithm has higher classification accuracy than the other methods. Although not satisfying, the obtained accuracies of the proposed methods for PaviaU data set are so close to the results obtained by the MSSC-MSF method [23]. Similarly, by comparing the result of our model and EPF [25], we can see that our result for Indian Pines is better than EPF [25], and for PaviaU, both results are so close. Moreover, in [28], the EPF [25] was compared with the single scale of our model (SSEPF-MMSF), and the results explain that for PaviaU, the SSEPF-MMSF works better [27].

We note that the obtained results of each models depend on the parameter setting and the researchers often insert the best parameters for the results [5, 6, 22-24].

Next, we demonstrate the marker selection process of MSF-based approaches [22, 23] from the point of view of time-consuming.

Marker selection is the main problem when an MSF is created. Even though showing a good performance for hyperspectral image analysis, using a marker-controlled approach of supervised segmentation is challenging due to the issue of marker selection. The SVM-MSF method [22] requires the computation of an SVM probabilistic map. In RD-MSF [24] and in the proposed methods since these methods do not need the probability estimates for selection of the markers, this time-consuming step is not used. The random selection of the markers in the proposed methods is at a higher speed compared to the marker selection done by connected component analysis [22]. The MSSC-MSF [23] approach is required to apply multispectral-spatial classifier in the marker selection step. Thus, the marker selection strategy is more time-consuming in comparison with the one that is used

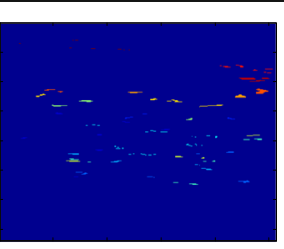

(a)

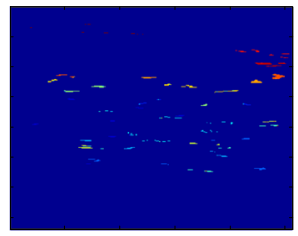

(b)

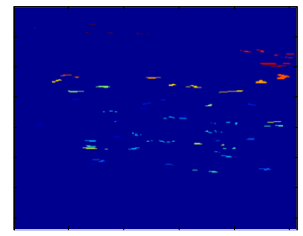

(c)

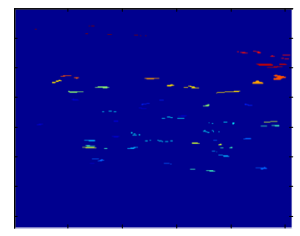

(d)

Fig. 11 Botswana image. a Ground truth data. b Pixel-wised classification map using SVM. c Classification map obtained by SSEPF-MMSF. d Classification map obtained by MSEPF-MMSF 
Table 6 Statistic of McNemar's test

\begin{tabular}{llllllll}
\hline Methods & \multicolumn{3}{l}{ SSEPF-MMSF } & & & & \\
\cline { 2 - 8 } & Scale \#1 & Scale \#2 & Scale \#3 & Scale \#4 & Scale \#5 & Scale \#6 & Scale \#7 \\
\hline MSEPF-MMSF & 3.41 & 2.32 & 2.14 & 2.45 & 2.65 & 4.36 & 4.26 \\
\hline
\end{tabular}

in the proposed approach. As a result, the suggested methods implement at less time than other MSF-based methods in the marker selection step.

\subsection{Second experiment}

In order to confirm the discussion of algorithm performance based on the provided parameter setting, the proposed algorithm (MSEPF-MMSF) is applied on two other well-known hyperspectral images including SalinasA and Botswana. In this experiment, the parameters of the algorithms are set at the optimal values extracted from the first experiment. In Tables 4 and 5, the number of training and test samples of each class and the classification accuracies for these data sets are given. In order to visualize the analysis, the obtained classification maps of the SVM classifier and the abovementioned classifiers (SSEPF-MMSF and MSEPF-MMSF) are given in Figs. 10 and 11. It is clear that the obtained classification map of MSEPF-MMSF is less noisy than the other two classification maps. Moreover, the classification accuracies are enhanced using the MSEPF-MMSF method. For example, the OA is improved by $6.65 \%$ and the AA by $6.43 \%$ when compared to the SVM classification for the Botswana data set. The result indicates that the good classification accuracies for the different images will be achieved with the use of the extracted optimal values of the parameters.

The following table represents McNemar's test [31] results for the Botswana data set (Table 6). The result of McNemar's test indicates the using of MSEPF-MMSF improves confidence of classification than SSEPFMMSF. MSEPF-MMSF will remove the statistical fluctuations of the classification. So, the efficiency and the robustness of the results will be enhanced. In fact, rather than preserving only the best classification result, the results of all classifiers even the unsuccessful ones are kept. The combination of these rich information using the majority voting decision rule provides the complementary spectral-spectral information and improves the classification accuracy. Thus, the confidence of classification will be improved. The results of the McNemar test for the Botswana image are given because it has a bigger size than the other researched images.

\section{Conclusions}

In this paper, a new method is proposed for spectralspatial classification by the combination of modified
MSF (MMSF) and multiscale edge-preserving filter (MSEPF). The proposed method can describe different spatial structures and performs faster than the traditional MSF because of the reduction in the number of nodes and edges. In addition, the marker-based approach of supervised segmentation which suffers from an issue of marker selection does not exist in this new method. The proposed method is also compared to some state-of-the-art methods. The results indicate that the proposed method has good performance in hyperspectral image classification. It is worth mentioning that the primary problem of the proposed method is determining the optimal values for the related parameters and that an automatic determination of these parameters would be a very interesting direction for future research.

\section{Acknowledgements \\ The authors would like to thank the management of the Department of Electrical Engineering, Science and Research Branch, Islamic Azad University, Tehran, Iran, for providing us with the infrastructure to complete the research work. \\ Funding \\ This work is supported by the Science and Research Branch of Islamic Azad University, Tehran, Iran.}

\section{Availability of data and materials}

Not applicable.

\section{Authors' contributions}

HG is the corresponding author. FP is the first author. Both authors made the algorithm and experiments. Both authors read and approved the final manuscript.

\section{Authors' information}

Hassan Ghassemian received his Electronics and Communication degree from Telecommunication College, Tehran, Iran, in 1980. He later got an M.E degree in Communication Engineering from the School of Electrical and Computer Engineering, Purdue University, USA, in 1984. He completed his PhD from the same university in the year 1988. His current research interests include Signal and Information Processing Multisource Image Analysis (Image and Information fusion), Pattern Recognition Remote Sensing System Engineering (Hyperspectral Image Analyses), and Biomedical Signal and Image Processing. He has published more than 100 research papers in reputed journals and conference proceedings. Fereshteh Poorahangaryan received her BE degree in Electronics Engineering from Amirkabir University, Iran, in 2006 and ME in Electronics Engineering from Guilan University, Iran, in 2009. She completed her PhD from the Science and Research Branch of Islamic Azad University, Iran, 2017. Her research interests include image processing, computer vision, and medical image analysis.

\section{Competing interests}

The authors declare that they have no competing interests.

\section{Publisher's Note}

Springer Nature remains neutral with regard to jurisdictional claims in published maps and institutional affiliations. 


\section{Author details}

'Department of Electrical Engineering, Science and Research branch, Islamic Azad University, Tehran, Iran. ${ }^{2}$ Image Processing and Information Analysis Lab. Faculty of Electrical and Computer Engineering, Tarbiat Modares University, Jalale Ale Ahmad Highway, P.O. Box 14115-111, Tehran, Iran.

Received: 8 June 2017 Accepted: 23 October 2017

Published online: 06 November 2017

\section{References}

1. C Yan, H Xie, D Yang, J Yin, Y Zhang, Q Dai, Supervised hash coding with deep neural network for environment perception of intelligent vehicles. IEEE Trans. Intell. Transp. Syst 99, 1-12 (2017). doi: 10.1109/TITS.2017.2749965

2. C Yan, Y Zhang, J Xu, F Dai, J Zhang, Q Dai, F Wu, A highly parallel framework for HEVC coding unit partitioning tree decision on many-core processors. IEEE Signal Process Lett 21(5), 573-576 (2014). doi: 10.1109/LSP.2014.2310494

3. C Yan, Y Zhang, J Xu, F Dai, J Zhang, Q Dai, F Wu, Efficient parallel framework for HEVC motion estimation on many-core processors. IEEE Trans. Circuits Syst. Video Technol 24(12), 2077-2089 (2014). doi: 10.1109/TCSVT.2014.2335852

4. X Ma, J Geng, H Wang, Hyperspectral image classification via contextual deep learning. EURASIP J Image Video Process 2015, 20 (2015). doi: 10.1186/s13640-015-0071-8

5. M Golipour, H Ghassemian, F Mirzapour, Integrating hierarchical segmentation maps with MRF prior for classification of hyperspectral images in a Bayesian framework. IEEE Trans Geos Remote Sens 54(2), 805-816 (2016). doi: 10.1109/TGRS.2015.2466657

6. A Zehtabian, $\mathrm{H}$ Ghassemian, An adaptive pixon extraction technique for multispectral/hyperspectral image classification. IEEE Geos. Remote Sens. Lett 12(4), 831-835 (2015). doi: 10.1109/LGRS.2014.2363586

7. L Zhang, D Tao, X Huang, Tensor discriminative locality alignment for hyperspectral image spectral-spatial feature extraction. IEEE Trans. Geos. Remote Sens 51(1), 242-256 (2013). doi: 10.1109/TGRS.2012.2197860

8. M Imani, H Ghassemian, Ridge regression-based feature extraction for hyperspectral data. Int. J. Remote Sens 36(6), 1728-1742 (2016). doi: 10.1080/01431161.2015.1024894

9. A Kianisarkaleh, $\mathrm{H}$ Ghassemian, Nonparametric feature extraction for classification of hyperspectral images with limited training samples. ISPRS J. Photogramm. Remote Sens 119, 64-78 (2016). doi: 10.1016/j.isprsjprs.2016.05.009

10. $Y$ Zhong, $L$ Zhang, An adaptive artificial immune network for supervised classification of multi-/hyperspectral remote sensing imagery. IEEE Trans Geos Remote Sens 50(3), 894-909 (2012). doi: 10.1109/TGRS.2011.2162589

11. HR Bittencourt, DA de Oliveira Moraes, V Haertel, in Proceedings of IEEE International Geoscience Remote Sensing Symposium. A binary decision tree classifier implementing logistic regression as a feature selection and classification method and its comparison with maximum likelihood (2007), pp. 1755-1758. doi: 10.1109/IGARSS.2007.4423159

12. G Camps-Valls, L Bruzzone, Kernel-based methods for hyperspectral image classification. IEEE Trans. Geos. Remote Sens 43, 1351-1362 (2005). doi: $10.1109 / T G R S .2005 .846154$

13. G Mountrakis, J Im, C Ogole, Support vector machines in remote sensing: a review. ISPRS J. Photogramm. Remote Sens 66(3), 247-259 (2011). doi: 10.1016/j.isprsjprs.2010.11.001

14. Q Jackson, D Landgrebe, Adaptive bayesian contextual classification based on Markov random fields. IEEE Trans Geos Remote Sens 40(11), 2454-2463 (2002). doi: 10.1109/TGRS.2002.805087

15. G Zhang, X Jia, Simplified conditional random fields with class boundary constraint for spectral-spatial based remote sensing image classification. IEEE Geos. Remote Sens. Lett 9(5), 856-860 (2012). doi: 10.1109/LGRS.2012.2186279

16. P Ghamisi, JA Benediktsson, MO Ulfarsson, Spectral-spatial classification of hyperspectral images based on hidden Markov random fields. IEEE Trans Geos Remote Sens 52(5), 2565-2574 (2014). doi: 10.1109/TGRS.2013.2263282

17. M Fauvel, Y Tarabalka, JA Benediktsson, J Chanussot, JC Tilton, Advances in spectral-spatial classification of hyperspectral images. Proc. IEEE 101(3), 652-675 (2013). doi: 10.1109/JPROC.2012.2197589

18. P Ghamisi, M Dalla Mura, JA Benediktsson, A survey on spectral-spatial classification techniques based on attribute profiles. IEEE Trans Geos Remote Sens 53(5), 2335-2353 (2015). doi: 10.1109/TGRS.2014.2358934
19. Y Tarabalka, J Chanussot, JA Benediktsson, Segmentation and classification of hyperspectral images using watershed transformation. Pattern Recogn 43(7), 2367-2379 (2010). doi: 10.1016/j.patcog.2010.01.016

20. Y Tarabalka, JA Benediktsson, J Chanussot, Spectral spatial classification of hyperspectral imagery based on partitional clustering techniques. IEEE Trans. Geosci. Remote Sens 47(9), 2973-2987 (2009). doi: 10.1109/TGRS.2009.2016214

21. Y Tarabalka, JC Tilton, JA Benediktsson, JA Chanussot, A marker-based approach for the automated selection of a single segmentation from a hierarchical set of image segmentations. IEEE J Sel Top Appl Earth Obs Remote Sens 5(1), 262-272 (2012). doi: 10.1109/JSTARS.2011.2173466

22. Y Tarabalka, J Chanussot, JA Benediktsson, Segmentation and classification of hyperspectral images using minimum spanning forest grown from automatically selected markers. IEEE Trans. Syst. Man Cybern. B Cybern 40(5), 1267-1279 (2010). doi: 10.1109/TSMCB.2009.2037132

23. Y Tarabalka, JA Benediktsson, J Chanussot, JC Tilton, Multiple spectral-spatial classification approach for hyperspectral data. IEEE Trans. Geosci. Remote Sens 48(11), 4122-4132 (2010). doi: 10.1109/TGRS.2010.2062526

24. A Kianisarkaleh, H Ghassemian, Spatial-spectral locality preserving projection for hyperspectral image classification with limited training samples. Int. J. Remote Sens 37(21), 5045-5059 (2016). doi: 10.1080/01431161.2016.1226523

25. X Kang, S Li, JA Benediktsson, Spectral-spatial hyperspectral image classification with edge-preserving filtering. IEEE Trans. Geosci. Remote Sens 52(5), 2666-2677 (2014). doi: 10.1109/TGRS.2013.2264508

26. JA Benediktsson, P Ghamisi, Spectral-spatial classification of hyperspectral remote sensing images (Artech House, Norwood, 2015)

27. F Poorahangaryan, $\mathrm{H}$ Ghassemian, Minimum spanning forest based approach for spatial-spectral hyperspectral images classification, 2016 Eighth International Conference on Information and Knowledge Technology (IKT) (2016), pp. 116-121. doi: 10.1109/KT.2016.7777749

28. K Bernard, Y Tarabalka, J Angulo, J Chanussot, JA Benediktsson, Spectralspatial classification of hyperspectral data based on a stochastic minimum spanning forest approach. IEEE Trans. Geosci. Remote Sens 21(4), 2008-2021 (2012). doi: 10.1109/TIP.2011.2175741

29. RD da Silva, H Pedrini, Hyperspectral data classification improved by minimum spanning forests. J. Appl. Remote. Sens 10(2), 025007 (2016). doi: $10.1117 / 1 . J R S .10 .025007$

30. H Song, Y Wang, A spectral-spatial classification of hyperspectral images based on the algebraic multigrid method and hierarchical segmentation algorithm. Remote Sens 8(4), 296 (2016). doi: 10.3390/rs8040296

31. GM Foody, Thematic map comparison: evaluating the statistical significance of differences in classification accuracy. Photogramm. Eng. Remote. Sens 70, 627-633 (2004). doi: 10.14358/PERS.70.5.627

\section{Submit your manuscript to a SpringerOpen ${ }^{\mathcal{O}}$ journal and benefit from:}

- Convenient online submission

Rigorous peer review

- Open access: articles freely available online

- High visibility within the field

- Retaining the copyright to your article

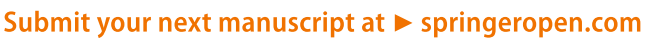

